\title{
Correlation study of relative parameters of intracranial pressure and prognosis of patient with craniocerebral injury
}

\author{
Laixing Liu ${ }^{1}$, Yixin Deng ${ }^{1}$, Fuquan Liu ${ }^{1}$, Lei Chen ${ }^{1}$, Dexi Li ${ }^{2}$, Xiangyi Wang*1 \\ ${ }^{1}$ Department of Neurosurgery, Baogang Hospital, Baotou, Inner Mongolia, China \\ ${ }^{2}$ Department of Medical Science, Inner Mongolia Medical University, Hohhot, Inner Mongolia, China
}

Received: March 28, 2020

DOI: $10.5430 /$ dcc.v6n4p20
Accepted: June 3, 2020

Online Published: June 14, 2020

\begin{abstract}
Objective: To analyze the correlation of the relative parameters of intracranial pressure to the prognosis in patients with craniocerebral injury.

Methods: The clinical data of 80 patients with closed craniocerebral injury were retrospectively analyzed, and all of these patients underwent conventional examinations of arterial blood pressure and intracranial pressure. Neumatic DCR system was used to monitor relative parameters of intracranial pressure from patients. According to the score of Glasgow outcome scale (GOS) upon discharge, they were divided into favorable prognosis group (GOS III-V, $\mathrm{n}=46$ ) and unfavorable prognosis group (GOS I-II, $\mathrm{n}=34$ ). The relative parameters of intracranial pressure of two groups were compared so as to analyze the correlation of the prognosis in patients to ICP-related parameters.

Results: Pressure reactivity index (PRx) and intracranial pressure (ICP) of favorable prognosis group were significantly higher than those of unfavorable prognosis group $(t=12.27, t=5.22, p<.05)$. Meanwhile, cerebral perfusion pressure $(\mathrm{CPP})$ and ICP-ABP wave amplitude correlation (IAAC) of favorable prognosis group were significantly lower than those of unfavorable prognosis group $(t=14.54, t=14.78, p<.05)$. The average age, gender, duration of admission to neurosurgical intensive care unit (NICU) and GCS (Glasgow coma scale) score on admission of the two groups were not statistically significant.

Conclusions: The prognosis and ICP-related parameters (such as PRx, ICP, CPP, etc.) in patients with craniocerebral injury are risk factors for the prognosis effect. Therefore, to monitor the above-mentioned indicators has an important clinical value for assessing the prognosis of craniocerebral injury.
\end{abstract}

Key Words: Influence factor, Prognosis, Pressure reactivity index, Continuous invasive ICP monitoring, Craniocerebral injury

\section{INTRODUCTION}

Intracranial pressure is defined as the pressure of craniocerebral contents against the wall of cranial cavity and represented by cerebrosinal fluid (CSF) pressure. Clinically, in the case that there is no obstruction in foramen magnum region and spinal subarachnoid space, it is usually represented by hydrostatic cerebrospinal fluid pressure measured in lumbar subarachnoid space, cerebellomedullary cistern and lateral ventricle. ${ }^{[1]}$ Currently, continuous invasive ICP monitoring is widely applied to the patients with severe craniocerebral China.

*Correspondence: Xiangyi Wang; Email: nmgbgyyyy@ 163.com; Address: Department of Neurosurgery, Baogang Hospital, Baotou, Inner Mongolia, 
injury in the aspect of clinical neurosurgery, in order to reduce the mortality of severe craniocerebral injury. Some researches have indicated that continuous invasive intracranial pressure (ICP) monitoring, ICP-arterial blood pressure wave amplitude correlation (IAAC), regression of amplitude and pressure (RAP), pressure reactivity index (PRx), cerebral perfusion pressure (CPP) and other ICP-related parameters can not only reflect a series of clinical indicators (e.g., intracranial brain compliance, intracranial compensatory space and intracranial vascular condition), but also predict the change in the prognosis and the degree of disease in patients. ${ }^{[2]}$ To this end, this study retrospectively analyzed the correlation of the prognosis to ICP-related parameters in 80 cases of patients with closed craniocerebral injury.

\section{DATA AND METHODS}

\subsection{General information}

A retrospective study was adopted to include 80 cases of patients who were diagnosed as closed craniocerebral injury in Baogang Hospital from June 2016 to April 2017. There were 46 cases of male patients and 34 cases of female patients at the age of 41-76 (averagely, 53.6 \pm 3.3), of which, 21 cases were injuries by falling, 26 cases resulted from traffic accidents, 21 cases were strike injuries and 12 cases resulted from other causes. On admission, Glasgow outcome scale (GOS) score was 3-13, with an average of $7.8 \pm 2.5$. According to GOS score upon discharge, these patients were divided into favorable prognosis group (GOS III-V, $\mathrm{n}=46$ ) and unfavorable prognosis group (GOS I-II, $n=34$ ). In favorable prognosis group, there were 26 cases of male patients and 20 cases of female patients at the age of 40-75 (averagely, $55.8 \pm 3.5$ ), of which, 11 cases were injuries by falling, 15 cases resulted from traffic accidents, 11 cases were strike injuries and 9 cases resulted from other causes. In unfavorable prognosis group, there were 20 cases of male patients and 14 cases of female patients at the age of 40-75 (averagely, 55.9 \pm 3.4 ), of which, 10 cases were injuries by falling, 11 cases resulted from traffic accidents, 10 cases were strike injuries and 3 cases resulted from other causes. It was comparable between two groups as the clinical information (e.g., average age, gender and pathogenic factors) from two groups of patients have no statistically significant difference. All subjects were approved by Ethics Committee of Baogang Hospital, and they all voluntarily participated in this study by signing informed consent forms.

\subsection{Inclusion and exclusion criteria}

\subsubsection{Inclusion criteria}

(1) Patients who complied with the diagnostic standards for acute closed craniocerebral injury established by WHO; ${ }^{[3]}$

Published by Sciedu Press
(2) Patients who were diagnosed by laboratory examinations, craniocerebral CT scanning and nuclear magnetic resonance imaging;

(3) Patients who were given external ventricular drainage in the emergency case;

(4) patients who had a clear history of trauma.

\subsubsection{Exclusion criteria}

(1) Patients who had underlying diseases such as hepatorenal failure and cardiopulmonary failure;

(2) Patients with coagulation abnormalities;

(3) Female patients during pregnancy and lactation;

(4) Patients with a history of epilepsy.

\subsection{Instruments and equipment}

MEC1000 ICP Patient Monitor (SOPHYSA, France); Neumatic DCR Data Acquisition System (Shanghai Haoju Medical Science and Technology Co., Ltd.).

\subsection{Treatment methods}

The statistical process was performed to all clinical information collected from all subjects, including GCS score on admission, age and gender. After admission to hospital, all patients were given RA puncture, ICP monitor insertion and ventricular puncture. Meanwhile, 40 cases of patients underwent decompressive craniectomy. According to operating procedures, Neumatic DCR system was then connected immediately after surgery to record and monitor all related indicators, including continuous ICP, IAAC, RAP, PRx and CPP. All patients were given neurosurgical standardized treatment.

\subsection{Evaluation indexes}

GOS and GCS scores were evaluated to compare ICP-related parameters between two groups, and Logistic regression method was used to analyze the influence factors affecting patients' prognosis and the correlation of the prognosis to ICP-related parameters.

\subsection{Statistical methods}

SPSS19.0 software was used to process and analyze all data. The categorical data were processed and tested by $\chi^{2}$. The measurement data fitted to normal distribution, and the comparison was made by use of $t$. The difference $p<.05$ was of statistical significance. Logistic regression method was used to analyze the prognosis in patients with craniocerebral injury and ICP-related parameters. GOS score upon discharge was used as a dependent variable, and patients' general information were independent variables. Sub-variables were set up for multiple categorical variables respectively. $p<$ .05 was considered as the stepwise regression standard for screening variables. 


\section{RESUlts}

\subsection{Analysis of the influence factors for GOS score}

$\mathrm{PRx}$ and ICP in favorable prognosis group were significantly higher than those in unfavorable prognosis group, the difference was of statistical significance $(t=12.27, t=5.22$; $p<.05$ ); the levels of CPP and IAAC in FP group were obviously lower than those in the other group, the difference was of statistical significance $(t=14.54, t=14.78 ; p<.05)$.
There was no statistically significant difference in gender, age, duration of admission to neurosurgical intensive care unit (NICU) and GOS score on admission of two groups. See Table 1 for details.

\subsection{Logistic regression analysis of the prognosis}

PRx, ICP and CPP proved to be risk factors for the prognosis of craniocerebral injury (see Table 2).

Table 1. Analysis of the influence factors for GOS score in the patients with craniocerebral injury (n)

\begin{tabular}{|c|c|c|c|c|c|c|c|c|c|c|c|}
\hline \multirow{2}{*}{ Group } & \multirow{2}{*}{$\mathbf{n}$} & \multicolumn{2}{|c|}{ Gender } & \multirow{2}{*}{ Age } & \multirow{2}{*}{ IAAC } & \multirow{2}{*}{ RAP } & \multirow[b]{2}{*}{ PRx } & \multirow{2}{*}{$\begin{array}{l}\text { CPP } \\
\text { (mmHg) }\end{array}$} & \multirow{2}{*}{$\begin{array}{l}\text { ICP } \\
\text { (mmHg) }\end{array}$} & \multirow{2}{*}{$\begin{array}{l}\text { Duration of } \\
\text { admission } \\
\text { to NICU (h) }\end{array}$} & \multirow{2}{*}{$\begin{array}{l}\text { GCS score } \\
\text { on } \\
\text { admission }\end{array}$} \\
\hline & & Male & Female & & & & & & & & \\
\hline $\begin{array}{l}\text { Favorable } \\
\text { prognosis } \\
\text { group }\end{array}$ & 46 & 26 & 20 & $\begin{array}{l}55.8 \pm \\
3.5\end{array}$ & $\begin{array}{l}0.05 \pm \\
0.01\end{array}$ & $\begin{array}{l}0.25 \pm \\
0.20\end{array}$ & $\begin{array}{l}0.11 \pm \\
0.07\end{array}$ & $\begin{array}{l}80.06 \pm \\
10.76\end{array}$ & $\begin{array}{l}13.94 \pm \\
5.73\end{array}$ & $\begin{array}{l}60.45 \pm \\
20.35\end{array}$ & $\begin{array}{l}8.39 \pm \\
3.18\end{array}$ \\
\hline $\begin{array}{l}\text { Unfavorable } \\
\text { prognosis } \\
\text { group }\end{array}$ & 34 & 20 & 14 & $\begin{array}{l}55.9 \pm \\
3.4\end{array}$ & $\begin{array}{l}0.12 \pm \\
0.03\end{array}$ & $\begin{array}{l}0.27 \pm \\
0.19\end{array}$ & $\begin{array}{l}0.50 \pm \\
0.20\end{array}$ & $\begin{array}{l}49.73 \pm \\
6.58\end{array}$ & $\begin{array}{l}26.19 \pm \\
14.47\end{array}$ & $\begin{array}{l}60.34 \pm \\
20.41\end{array}$ & $\begin{array}{l}8.23 \pm \\
3.02\end{array}$ \\
\hline$t$ Value & & 0.04 & & 0.13 & 14.78 & 0.45 & 12.27 & 14.54 & 5.22 & 0.02 & 0.23 \\
\hline$p$ Value & & .84 & & .90 & $<.01$ & .65 & $<.01$ & $<.01$ & $<.01$ & .98 & .82 \\
\hline
\end{tabular}

Note. IAAC: ICP-ABP wave amplitude correlation; RAP: regression of amplitude and pressure; PRx: pressure reactivity index; CPP: cerebral perfusion pressure; ICP: intracranial pressure; NICU: neurosurgical intensive care unit; GCS: Glasgow coma scale (mild coma rates 13 to 14, moderate coma rates 9 to 12 and severe coma rates 3 to 8 ).

Table 2. Logistic regression analysis results that affecting the prognosis in the patients with craniocerebral injury

\begin{tabular}{llllllll}
\hline Influence Factors & $\boldsymbol{\beta}$ & SE & Wald & Df & $\boldsymbol{p}$ Value & Exp ( $\boldsymbol{\beta})$ & $\mathbf{9 5 \%}$ CI \\
\hline PRx & 0.890 & 0.583 & 18.683 & 1 & .000 & 2.683 & $1.217-3.489$ \\
CPP & 1.796 & 1.573 & 12.754 & 1 & .002 & 3.387 & $1.609-5.932$ \\
ICP & 1.477 & 1.682 & 20.758 & 1 & .000 & 2.286 & $1.743-3.854$ \\
\hline
\end{tabular}

Note. B: partial regression coefficient; SE: standard error; Wald: chi-square value; Df: degree of freedom; Exp ( $\beta$ ): the index of the coefficient $\beta ; 95 \%$ CI: confidence interval; PRx: pressure reactivity index; CPP: cerebral perfusion pressure; ICP: intracranial pressure.

\section{Discussion}

Craniocerebral injury is a clinically commonly seen neurosurgical emergency. Among urban death and injury cases in China, about $60 \%$ of them result from craniocerebral in jury. ${ }^{[4,5]}$ Craniocerebral injury consists of 3 types: scalp injury, skull injury and brain injury. They usually occur in combination or alone. For patients with severe craniocerebral injury (i.e., patients with diffuse brain injury as well as patients with ICP exceeding $20 \mathrm{mmHg}$ in one hour after injury for more than $15 \mathrm{~min}$ and no response to the first-line treatment), bilateral decompressive craniectomy can reduce ICP and shorten the length of stay in ICU. ${ }^{[6]}$ Simultaneously, it is required to perform preventive mild hypothermia therapy, high-permeability treatment, cerebrospinal fluid drainage, nutritional support, infection-preventing treatment, epilepsy-preventing treatment and ICP monitoring etc.. The information acquired from ICP monitoring can be used to administrate the patients with severe craniocerebral injury in order to reduce the length of stay and the mortality in 2 weeks after surgery. For the patients with severe craniocerebral injury showing normal CT scanning results on admission, it is recommended to perform ICP monitoring if more than two of the following conditions are met: systolic pressure $<90$ $\mathrm{mmHg}$, bilateral or unilateral limb movement disorder and age $>40$. It is strongly recommended to perform a symptomatic treatment to the patients whose ICP is higher than $22 \mathrm{mmHg}$, because exceeding the threshold value is closely associated with the increase of the mortality. Clinically, it is also required to take clinical symptoms, ICP values and craniocerebral CT scanning results into account to build up a basis for the decision-making of the clinical data. ${ }^{[7]}$

Currently, the study on the prognosis in patients with craniocerebral injury and ICP-related parameters has been a topic of intense interest in the medical field. ${ }^{[8]}$ The research results show that PRx and ICP in favorable prognosis group are significantly higher than those in unfavorable prognosis group; 
however, the level of CPP in the former group is obviously lower than that in the latter group. There is no statistically significant difference in the average age, gender, duration of admission to NICU and GCS score on admission; PRx, ICP and CPP prove to be risk factors for the prognosis of craniocerebral injury, which basically conforms to the research results acquired by Liansheng Long et al. ${ }^{[9]}$ Clinically, the increase of ICP indicates ICP is over $15 \mathrm{mmHg}$. In the light of the correlation curve of intracranial volume and pressure, when a patient's ICP exceeds a certain critical point, if the intracranial volume slightly increases, it will lead to a significant increase in ICP. ${ }^{[10]}$ For the patients with craniocerebral injury, cerebrovascular autoregulation has fallen into loss of function. The response of ICP to the change in arterial blood pressure depends on cerebrovascular reactivity, which is the main component of cerebrovascular autoregulation. The impairment of cerebrovascular reactivity indicates the destruction of cerebrovascular autoregulation, and PRx can be acquired by continuously monitoring and analyzing the slow wave of ABP and ICP. ${ }^{[11,12]}$ PRx refers to linear correlation coefficient of ICP and $\mathrm{ABP}$, and it ranges from - 1 to 1. When cerebrovascular reactivity is at a normal level, the rise of $\mathrm{ABP}$ can result in cerebral vasoconstriction in 5-15 s, with cerebral blood flow and ICP decreasing consequently, vice versa. ICP and ABP values on uniform time points are required to be continuously monitored and recorded within 4 min to calculate the correlation coefficient in order to acquire PRx. ${ }^{[13,14]}$ When PRx is negative, i.e., ABP is negatively correlated to ICP, it indicates that cerebrovascular reactivity is at a normal level; when PRx is positive, it indicates that cerebrovascular reactivity is impaired. The research results show that PRx and ICP in favorable prognosis group are significantly higher than those in unfavorable prognosis group. After craniocerebral injury occurs, higher PRx indicates a worse prognosis. It is feasible to continuously monitor PRx, which has been applied to the individualized treatment target for specific CPP after cerebral trauma. Pressure-volume compensatory space, i.e., the relationship of ICP and cerebral blood flow, can be used to define the index of compensatory space (i.e., regression of amplitude and pressure, RAP). RAP refers to the relationship of pulse amplitude and the mean ICP monitored within 1-3 min, and it ranges from -1 to +1 . When the ICP-volume curve becomes flat, the pulse amplitude is not synchronous with the change in ICP, which represents a good compensatory space. At this moment, RAP is equal to 0 , with a lower amplitude of ICP. When the curve becomes steep, the compensatory space becomes smaller, and pulse amplitude directly changes along with ICP, and RAP is equal to +1 . The amplitude of ICP is increased with the rise of the mean ICP slowly at the first beginning. With the exhaustion of the compensatory space, the amplitude becomes larger quickly. At the end of the curve, if RAP is lower than 0 , the impairment of final cerebrovascular reactivity will happen. The pulse pressure from the arterial bed decreases to intracranial pressure and forms a low intracranial pressure wave, even worse, shows no intracranial pressure wave. If RAP is lower than 0.5 and ICP is more than $20 \mathrm{mmHg}$, it indicates an unfavorable prognosis of craniocerebral injury.

It is also found in this research that, the levels of CPP and IAAC in FP group are significantly lower than those in UP group. The multi-parameter monitoring of ICP and CPP in combination with cerebral oxygenation and metabolism monitoring can more completely present the condition after craniocerebral injury and comprehensively reflect the treatment effect. Some researches indicate that, among the patients with craniocerebral injury, if the lower limit of CPP is maintained at $70 \mathrm{mmHg}$, they are 5 times more susceptible to pulmonary injury than those whose lower limit of CPP is maintained at $50 \mathrm{mmHg}$. It indicates hyper-perfusion treatment may result in more severe complications. The loss of cerebrovascular autoregulation and the increase of CPP can lead to cerebral stroke, aggravate vasogenic cerebral edema and result in secondary increased ICP. Some researches also show that the target of CPP for severe craniocerebral injury should be controlled in the range of $50-70 \mathrm{mmHg}$.

The prognosis and ICP-related parameters (such as PRx, ICP, CPP etc.) in patients with craniocerebral injury are risk factors for the prognosis effect. Therefore, to monitor the above-mentioned indicators has an important clinical value for assessing the prognosis of craniocerebral injury.

\section{CONFLICTS OF INTEREST DisClosure}

The authors declare they have no conflicts of interest.

\section{REFERENCES}

[1] Kochanek PM, Jackson TC, Jha R, et al. Paths to successful translation of new therapies for severe TBI in the golden age of traumatic brain injury research: a pittsburgh vision. J Neurotrauma. 2018; 27(6): 5506-5506, 5507.

Published by Sciedu Press
[2] Jianhua Zhao, Jun Zhu, Chao Wang, et al. Effect of continuous reduction of intracranial pressure on the prognosis of patients with severe traumatic brain injury. Chinese Journal of Medicine. 2018; 53(9): 1000-1002.

[3] Caigan Liang, Zhen-xia Li, Da-an Qin, et al. Study on the application 
of ICP-monitoring guided stepwise therapy to the patients with moderate craniocerebral injury. China Practical Medicine. 2018; 13(29): 43-45.

[4] Zhitong Han, Ruijian Zhang, Yunzhao Chen, et al. Application of intracranial pressure combined with cerebral blood flow monitoring after standard bone flap decompression for patients with severe craniocerebral injury. China Medical Herald. 2018; 15(23): 52-55.

[5] Yuzhang Yan. Comparison of the curative effect of standard large bone flap decompression and conventional decompressive craniectomy in the treatment of severe craniocerebral injury. China Modern Doctor. 2018; 56(23): 81-83.

[6] Jian Huang. Clinical research of disease prognosis judgment and treatment guidance by intracranial pressure monitoring for moderate and severe craniocerebral injury patients. China Practical Medicine. 2017; 12(18): 4-5.

[7] Xiang Wu, Guoyi Gao, Junfeng Feng, et al. Analysis of correlation between intracranial pressure and outcome in traumatic brain injury patients. Chinese Journal of Neurosurgery. 2018; 34(2): 119-123.

[8] Qibing Huang, Yuan Zhang, Yuhang Su, et al. Prognostic correlation of intracranial pressure monitoring in patients with severe craniocerebral injury. National Medical Journal of China. 2013; 93(23): 1788-1790.
[9] Liansheng Long, Wei Wang, Cong Wang, et al. Effect of the combination of transcranial Doppler and invasive intracranial pressure monitoring in the treatment of post-traumatic bilateral cerebral hemisphere diffuse brain swelling patients. Chinese Journal of Critical Care Medicine (Electronic Edition). 2016; 9(5): 289-293.

[10] Portnova GV, Atanov MS. EEG of patients in coma after traumatic brain injury reflects physical parameters of auditory stimulation but not its emotional content. Brain Inj. 2018; 12(6): 1-7.

[11] Guangping Zhang, Haiming Shen, Shutao Gao, et al. Study on the correlation of the prognosis of sTBI to ICP, BT and EEG. Chinese Journal of Neurosurgery. 2015; 31(4): 374-375.

[12] Qiping Wang, Shiming Zhang, Heng Gao, et al. Guiding value of brain tissue oxygenation plus intracranial pressure monitoring in severe traumatic brain injury patients. National Medical Journal of China. 2013; 93(23): 1784-1787.

[13] Liang Gao. Evaluation of the role of ICP monitoring in the treatment of severe traumatic brain injury. Chinese Journal of Trauma. 2013; 29(2): 100-102.

[14] Neurocritical Collaborative Group of Chinese Society of Neurology of Chinese Medical Association, Neurocritical Specialized Committee of Neurological Physician Society of Chinese Medical Doctor Association. Monitoring and treatment of refractory increased ICP Chinese expert consensus. National Medical Journal of China. 2018; 98(45): 3643-3652. 